\title{
Metal Nanocatalysts in Solution: Characterization and Reactivity
}

\author{
Bruno Chaudret $\cdot$ Montserrat Gómez $\cdot$ \\ Karine Philippot
}

Published online: 1 August 2013

(c) Springer Science+Business Media New York 2013

Homogeneous catalysis has emerged as a very important field thanks to the high selectivities reached nowadays by these catalysts and to the molecular understanding of the reactions involved. Heterogeneous catalysis continues to be of very high industrial importance and will be a major actor in the energy transition. Present trends in this field concern the precise monitoring of molecular surface reactivity.

Colloidal catalysis, which remained marginal for a long time, has gained more and more interest. Thus it concerns reactions typical of molecular chemistry such as carboncarbon or carbon-heteroatom coupling. One major goal in this field is to reach enantioselectivity comparable to the molecular systems. However this remains a challenge although some novel approaches become available, such as the ones described in this issue. Colloidal catalysis also

Guest editors: Bruno Chaudret, Montserrat Gómez, Karine Philippot

B. Chaudret $(\bowtie)$

Laboratoire de Physique et Chimie de Nano-Objets (LPCNO), UMR 5215 INSA-CNRS-UPS, Institut National des Sciences Appliquées, 135 Avenue de Rangueil, 31077 Toulouse, France e-mail: chaudret@insa-toulouse.fr

\section{Gómez}

Laboratoire Hétérochimie Fondamentale et Appliquée, UMR CNRS 5069, Université Paul Sabatier, 118 Route de Narbonne, 31062 Toulouse cedex 9, France

e-mail: gomez@chimie.ups-tlse.fr

K. Philippot

CNRS, Laboratoire de Chimie de Coordination (LCC), 205 Route de Narbonne, 31077 Toulouse, France

e-mail: karine.philippto@lcc-toulouse.fr

K. Philippot

Université de Toulouse, UPS, INPT, LCC, 31077 Toulouse,

France allows access to reactions that standard molecular chemistry cannot achieve such as arene hydrogenation or Fischer-Tropsch synthesis and addresses the important field of energy (Fischer-Tropsch synthesis again and hydrogen generation). Colloidal catalysis is also part of the tendency of chemistry to "go green", nanoparticles may be soluble in water or in ionic liquids which offer the advantage of resembling supported catalysis while allowing facile heat diffusion.

The synthesis of nanoparticles has progressed tremendously for the past 20 years and now it is possible to prepare controlled species, mono or bimetallic, either with or without stabilizers at the metallic surface (such as ligands, salts, polymers, hydrides, $\mathrm{CO}$, and so on), which eventually may be deposited on a support to give rise to well-defined heterogeneous catalysts. These species may expose a defined surface, a controlled chemical order or a number of ordered surface ligands and have given rise to the concept of "Nanocatalysis." Spectroscopic investigations are carried out in order to lead to a molecular description of the surface reactivity, in particular through the development of more and more precise NMR methods while the chemical understanding of surface reactivity today benefits from the impressive progresses in DFT modeling which can now use ab initio methods.

This issue of Topics in Catalysis profits from the diverse approaches described here above to offer a snapshot of the present tendencies in this important field. 